\title{
Akademievorträge
}

\section{Stammzellforschung - eine wissenschaftliche und politische Gratwanderung}

(Festvortrag in der öffentlichen Jahresfeier am 20. November 2010)

\section{Gerd Hasenfuss}

Gesetzliche Rahmenbedingungen der Forschung

Wohl kaum ein Forschungsgebiet wird von der Öffentlichkeit so intensiv wahrgenommen wie die Stammzellforschung. Für die einen stellt sie die Möglichkeit dar, grundlegend neue Behandlungsverfahren in der Medizin zu entwickeln, von anderen wird sie verteufelt und mit der Abtötung menschlichen Lebens assoziiert. Wohl kaum ein Forschungsgebiet wurde im Gesetzgebungsverfahren eingehender analysiert als die Stammzellforschung, und kein Forschungsgebiet wurde in Deutschland

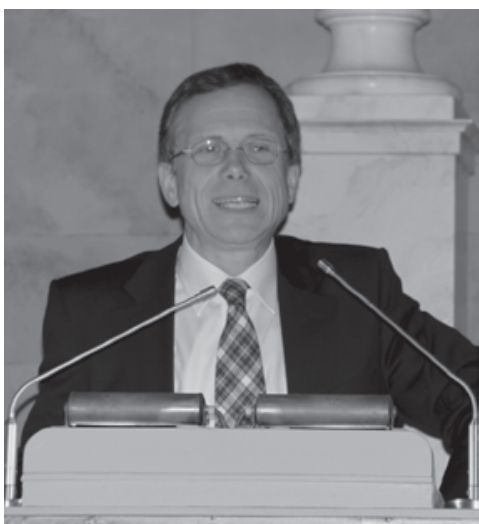

Gerd Hasenfuss, Professor für Innere Medizin an der Georg-August-Universität Göttingen, O. Mitglied der Göttinger Akademie seit 2002 vom Gesetzgeber so einschneidend reglementiert. Nie - vermutlich - wurde ein Gesetzgebungsverfahren eingehender von wissenschaftlichen Interessen beeinflusst, und nie zuvor hat die Interaktion zwischen Wissenschaft, öffentlicher Debatte und gesetzlicher Regelung die Entwicklung der Forschung stärker beeinflusst als in der Stammzellforschung. Im folgenden soll daher die Wechselwirkung zwischen den gesetzlichen Rahmenbedingungen und der Forschungsentwicklung aufgearbeitet und soll verdeutlicht werden, wie entscheidend die gesellschaftliche Debatte, die Konsensfindung und schließlich die Gesetzgebung für die Forschung und den medizinischen Fortschritt sein können. 


\section{Das Embryonenschutzgesetz}

Das Embryonenschutzgesetz wurde im Dezember 1990 verabschiedet (1) ${ }^{1}$. Es ist mit dem Ziel entwickelt worden, die in-vitro-Fertilisation zu regeln. Es definiert den Beginn menschlichen Lebens unter der Bezeichnung „Embryo" mit der Befruchtung einer Eizelle. Auf nicht-natürlichem Wege in vitro - dürfen danach Embryonen nur zum Zwecke der Fortpflanzung erzeugt werden. Eine Befruchtung zu einem anderen Zwecke als zum Erreichen einer Schwangerschaft ist strafbar. Überzählige Embryonen sollen im Rahmen der extrakorporalen Befruchtung nicht entstehen und dürfen nicht verwendet werden.

Das Embryonenschutzgesetz in Deutschland und seine Analoga in anderen Ländern bedeuteten weltweit ein wesentliche Limitierung für die Stammzellforschung, eine Wissenschaftsrichtung in der Biologie und Medizin, von der viele glauben, dass sie die zweite lebensverlängernde Revolution in der Medizin nach der Einführung von Antibiotika im vergangenen Jahrhundert bringen werde.

Welche Beziehung besteht zwischen einem Embryo und einer Stammzelle? Die embryonale Stammzelle entstammt einem Embryo, genau genommen, dessen innerer Zellmasse, der so genannten Blastozyste im 32-Zellen-Stadium. Dieser Reifezustand ist vier bis fünf Tage nach der Befruchtung erreicht. Es handelt sich also um ein frühes Embryonalstadium. Die Gewinnung von embryonalen Stammzellen führt zur Zerstörung des Embryos.

\section{Definition der Stammzelle und der Pluripotenz}

Unter einer Stammzelle versteht man jede unspezialisierte Zelle eines Organismus, die sich teilen und vermehren und dann reife Organzellen bilden kann. Die organspezifische Spezialisierung der aus einer Stammzelle entstandenen Tochterzelle wird als Differenzierung bezeichnet. Wird also eine unspezialisierte Stammzelle zu einer Herzzelle oder einer Nervenzelle, so wird dies als Differenzierung bezeichnet. Pluripotenz bedeutet, dass eine Stammzelle in alle spezifischen Organzellen des Körpers - das sind mehr als 200 verschiedene Zelltypen - differenzieren kann. Multipotent bedeutet dementsprechend, dass die Stammzelle in viele, aber nicht alle Gewebetypen differenzieren kann. Die Pluripotenzeigenschaften hat man zunächst nur embryonalen Stammzellen zugeschrieben. Im Gegensatz zur embryonalen

$\overline{1}$ Siehe auch http://www.ethikrat.org/dateien/pdf/stellungnahme_stammzellimport.pdf. 
Stammzelle entstammt eine adulte Stammzelle dem Erwachsenenorganismus. Adulte Stammzellen sind in ihrem Differenzierungspotential häufig eingeschränkt. Embryonale Stammzellen sind also „Alleskönner“, adulte Stammzellen aus dem Erwachsenenorgan häufig nur „Vielkönner“.

\section{Die Bedeutung von Stammzellen für die Medizin}

Stammzellen wird im Rahmen der regenerativen Medizin eine erhebliche Bedeutung für die Therapie von Organerkrankungen beigemessen. Darüber hinaus können Stammzellen in erheblichem Umfang dazu beitragen, dass Organerkrankungen verstanden und stammzellenunabhängige Behandlungsformen entwickelt werden. Hierfür ein Beispiel: Nach einem Herzinfarkt entsteht eine Narbe, und fast 30\% aller Patienten mit Herzinfarkt entwickeln eine Herzmuskelschwäche, die so genannte Herzinsuffizienz. Dies ist darauf zurückzuführen, dass das hochspezialisierte Herzmuskelgewebe die Fähigkeit verloren hat, in adäquatem Umfang neue Herzmuskelzellen nachzubilden. Daher kann abgestorbenes Herzgewebe nach einem Herzinfarkt nur durch Narbengewebe, aber nicht durch neues Herzmuskelgewebe ersetzt werden. Anders verhält es sich beim Blut. Hier befinden sich im Knochenmark blutbildende Stammzellen, die sich nach Blutverlust teilen und eine folgenlose Heilung gewährleisten. Auch andere Organe wie die Haut, die Leber und die Skelettmuskulatur besitzen ein gewisses Regenerationspotential. Demgegenüber können Untergänge von Nervenzellen bei Schlaganfall oder bei der Querschnittslähmung - vergleichbar dem Herzmuskelzelluntergang - nicht adäquat regeneriert werden. Ähnliches gilt für die insulinproduzierenden Bauchspeicheldrüsenzellen, nach deren Untergang ein Diabetes mellitus entsteht. Pluripotente Stammzellen könnten also in Herz-, in Nerven- oder in Bauchspeicheldrüsenzellen ausdifferenzieren und dann zur Regeneration nach Herzinfarkt, Schlaganfall oder bei Diabetes eingesetzt werden. Kritisch anzumerken bleibt, dass vielfach postuliert wurde, man werde unter Verwendung von Stammzellen innerhalb von wenigen Jahren risikoarme Therapieverfahren entwickeln können. Dabei nahm man an, dass man die Stammzellen in das erkrankte Organ injizieren, also transplantieren könne und dass diese dann in spezialisierte Organzellen ausdifferenzieren würden (2). Diese Hoffnung der ersten Stunden der Stammzellforschung ist nicht in Erfüllung gegangen (3). Bei der Transplantation von Stammzellen kann es im Organ zu Abstoßungsreaktionen kommen, auch dann, wenn die Stammzellen aus dem eigenen Organismus kommen, und erst recht, wenn immunologisch fremde embryonale Stammzellen verwendet werden. Hier müssen 
also Techniken zur Überwindung der Körperabwehr entwickelt werden. Ferner können Stammzellen nach Transplantation Tumore ausbilden (4). Die Verwendung von pluripotenten Stammzellen in der Organregeneration wird also nur dann möglich sein, wenn Abstoßungs- und Tumormechanismen verstanden und die Tumorbildung mit einem hohen Maß an Sicherheit ausgeschlossen werden können. Vor kurzem ist in den USA bei einem Patienten mit akuter Querschnittslähmung die erste Therapie mit Zellen durchgeführt worden, die aus embryonalen Stammzellen gewonnen worden waren. Die Zellen wurden am Ort der Schädigung ins Rückenmark injiziert, und man hofft, dass sie dafür sorgen werden, dass sich die durchtrennten Nervenfasern wieder verbinden. Die amerikanische Zulassungsbehörde FDA hatte diesen Heilversuch genehmigt, nachdem in vorangehenden Affenexperimenten keine Komplikationen aufgetreten waren (5).

Ein zweites potentielles Einsatzgebiet der pluripotenten Stammzellen ist die Verwendung der Zellen zur Erforschung von Organerkrankungen und zur Entwicklung neuer Behandlungsverfahren (s. u.).

\section{Embryonenschutzgesetz, Stammzellgesetz und Stichtagsregelung}

Warum hat das Embryonenschutzgesetz von 1990 die medizinische Bedeutung der Stammzellen nicht berücksichtigt? Weil menschliche embryonale Stammzellen erst 1998 isoliert wurden. 1981 wurden embryonale Stammzellen erstmals erfolgreich aus Mäuseembryonen kultiviert. Es dauerte dann 17 Jahre, bis 1998 die Amerikaner Thomsen und Mitarbeiter erfolgreich menschliche embryonale Stammzellen kultivieren konnten (6). Sie isolierten diese Zellen aus einer menschlichen Blastozyste, die aus der extrakorporalen Befruchtung übriggeblieben und gespendet worden war. Unter dem Embryonenschutzgesetz von 1990 war also die Forschung an menschlichen embryonalen Stammzellen in Deutschland gesetzeswidrig. Hieraus entwickelte sich in Deutschland eine sehr intensive und emotional geführte Debatte pro versus contra embryonale Stammzellforschung. Unter dem Druck der Wissenschaftler, aber wohl auch unter der Vorstellung, in anderen Ländern könnten mit solchen Zellen neue Behandlungsverfahren entwickelt werden, die dann deutschen Patienten nicht würden vorenthalten werden können, stellte der Deutsche Bundestag am 29.01.2002 fest: ${ }^{2}$ „Menschliche embryonale Stammzellen sind jedoch keine Embryonen, weil sie sich nicht zu einem vollständigen menschlichen Organismus entwickeln können. Ein unmittelbarer Grundrechtschutz kann für sie nicht in Anspruch genom-

$2 \quad$ Deutscher Bundestag, Drucksache 14/8102 
men werden. Deshalb stehen dem Grundrecht der Freiheit der Wissenschaft und Forschung, dessen Schranken sich nur aus der Verfassung selbst ergeben können, bei der Forschung an humanen embryonalen Stammzellen keine unmittelbar kollidierenden Grundrechte eines Embryos entgegen". Man hat daher darüber debattiert, ob der Import von embryonalen Stammzellen aus dem Ausland für die Forschung gestattet werden könne, allerdings nur, wenn solche Zellen schon als Einzelzellen in Kultur vorliegen und nicht mehr erst aus einem Embryo gewonnen werden müssen mit der Folge, dass dieser abstirbt. Es heißt dann weiter: „Gleichwohl ist der Import von humanen embryonalen Stammzellen rechtlich und ethisch problematisch, da ihre Gewinnung nach derzeitigem Stand von Wissenschaft und Technik die Tötung von Embryonen voraussetzt. Deshalb muss sichergestellt werden, dass der Import von humanen embryonalen Stammzellen nach Deutschland keine Tötung weiterer Embryonen zur Stammzellgewinnung veranlasst."

Diese Überlegungen bildeten die Grundlage für das Stammzellgesetz vom 28. Juni 2002 (7). Danach dürfen zu Forschungszwecken menschliche embryonale Stammzellen verwendet werden, aber nur solche, die im Ausland erzeugt worden sind. Zugleich mussten diese Stammzellen vor dem 1. Januar 2002 entstanden sein und seither in Kultur gehalten werden. Die embryonalen Stammzellen müssen aus Embryonen stammen, die aus der extrakorporalen Befruchtung zum Zwecke der Herbeiführung einer Schwangerschaft erzeugt worden sind und nicht mehr benötigt werden. Es darf kein Entgelt oder sonstiger geldwerter Vorteil gewährt worden sein. Forschungsvorhaben müssen eingehend begründet und einem intensiven Prüfungsverfahren unterzogen werden. Herr des Verfahrens ist das RobertKoch-Institut. Das Forschungsprojekt muss wissenschaftlich hochwertig und auf die Verwendung menschlicher embryonaler Stammzellen angewiesen sein.

Die gesetzliche Situation war von Land zu Land verschieden. Die embryonale Stammzellforschung war in England oder in Israel deutlich weniger oder gar nicht reglementiert. In USA gab es unter der Bush-Regierung zwar kein Verbot der Forschung mit menschlichen embryonalen Stammzellen aus überflüssigen Embryonen, aber öffentliche Gelder wurden für diese Art von Forschung nicht zur Verfügung gestellt. Unter Obama wird auch die Forschung an embryonalen Stammzellen wieder finanziell gefördert.

Im Jahre 2007 begannen die Embryonenschutzdebatte und die Debatte um die Stichtagregelung erneut. Grund hierfür war, dass nunmehr die Stammzellen, die aus dem Ausland für die Forschung in Deutschland importiert werden durften, mindestens fünf Jahre alt waren - die Stichtags- 
regelung besagte ja, dass die Zellen vor dem 1. Januar 2002 entstanden sein und in Kultur gehalten werden mussten. Mit derartigen Zellen waren keine vernünftigen wissenschaftlichen Ergebnisse mehr zu erzielen, geschweige denn Behandlungsverfahren zu entwickeln und durchzuführen. Die Stichtagsregelung wurde daher abgeändert.

Am 11.4.2008 stimmt der Bundestag für eine Stichtagsänderung im Stammzellgesetz. „In namentlicher Abstimmung votierten 346 Abgeordnete für eine einmalige Verschiebung des Stichtags für zur Forschung freigegebene Stammzellen auf den 1. Mai 2007. Dagegen stimmten 228 Parlamentarier, 6 enthielten sich. Zuvor waren im Parlament sowohl ein Vorstoß zur völligen Abschaffung der Stichtagsregelung als auch ein Gesetzentwurf zum Verbot der Forschung mit menschlichen embryonalen Stammzellen gescheitert. Vorausgegangen war eine emotionale Debatte, in der die unterschiedlichen Meinungen aufeinander prallten. Die Fronten reichten quer durch die Parteien. Es wurde daher ohne den sonst üblichen Fraktionszwang abgestimmt". 3

\section{Definition des Lebensbeginns}

Warum wird diese Debatte in ihrer Neuauflage erneut so kontrovers über die gesamte Breite der gesetzlichen Möglichkeiten hoch emotional geführt? Ein Grund hierfür liegt in der Definition des Lebensbeginns. Menschliches Leben beginnt nach deutschem Gesetz bereits mit der befruchteten entwicklungsfähigen Eizelle. Die Eizelle entwickelt sich innerhalb der ersten fünf Tage nach der Befruchtung zur Blastozyste, die die embryonalen Stammzellen enthält. Zu diesem Zeitpunkt befindet sich die Blastozyste noch nicht in der Gebärmutter, die Implantation erfolgt am sechsten Tage. Vor der Implantation ist der Embryo allein nicht lebensfähig. Für die weitere Entwicklung ist die Umgebung der Gebärmutter mit den dort freigesetzten Wachstumssubstanzen conditio sine qua non. Man hätte sich also durchaus auch für den Zeitpunkt der Implantation oder Nidation des Embryos in der Gebärmutter als Lebensbeginn entscheiden können. Entsprechend gibt es in anderen Kulturen und Religionen abweichende Definitionen des Lebensbeginns. So liegt nach jüdischem Glauben der Lebensbeginn am 40. Tage nach der Befruchtung. Daher ist in Israel die Forschung mit embryonalen Stammzellen erlaubt, die fünf Tage nach der Verschmelzung gewonnen werden.

3 http://www.stammzellen-debatte.de/stammzellen_news_11-04-08-pressespiegel-3.html. 


\section{Die Entwicklung der Stammzellforschung}

Wie konnte sich nun die Stammzellforschung unter den gesetzlichen Rahmenbedingungen entwickeln? Unter dem Druck der Forschungsrestriktionen und unter der Erkenntnis, dass selbst bei einer erfolgreichen Entwicklung von therapeutischen Verfahren der Einsatz von embryonalen Stammzellen zur Behandlung von Erkrankungen womöglich wissenschaftlich und politisch nicht akzeptiert werden würde, haben sich viele Wissenschaftler mit der Erforschung von nichtembryonalen Stammzellen auseinandergesetzt. Dies wurde in Deutschland unterstützt durch Fördermittel des Bundes. Hierbei geht es um die Suche nach - pluripotenten - Stammzellen aus dem Erwachsenenorganismus, mit dem Ziel, auf menschliche embryonale Stammzellen langfristig verzichten zu können.

Um dieses Ziel erreichen zu können, muss man aber die Eigenschaften der embryonalen Stammzellen kennen. Ein zukünftiger Verzicht auf embryonale Stammzellen war also nur aussichtsreich, wenn Forschung an menschlichen embryonalen Stammzellen durchgeführt werden konnte. Ein Verbot der Forschung mit embryonalen menschlichen Stammzellen hätte die Entwicklung von pluripotenten adulten Stammzellen verhindert.

\section{Die spermatogoniale Stammzelle}

Im Jahr 2006 ist es gelungen, mit der spermatogonialen Stammzelle der Maus eine adulte pluripotente Stammzelle des Erwachsenenorganismus zu identifizieren $(8,9)$. Dabei war bekannt, das Keimbahnzellen im embryonalen Stadium oder unmittelbar nach der Geburt pluripotent sein können. Es war bisher allerdings postuliert worden, dass dies im Erwachsenenzustand nicht mehr zutreffe. Eine dieser Keimbahnzellen ist die spermatogoniale Stammzelle. Sie ist im Hoden für die lebenslang dauernde Spermienproduktion verantwortlich. Sie teilt sich, und während eine Tochterzelle Stammzelleigenschaften behält, differenziert die andere unter Halbierung des Chromosomensatzes zu einem Spermium. Gegenwärtig wird an der Etablierung der Technik für menschliches Gewebe gearbeitet. Auch hier gilt, dass auf die Forschung an menschlichen embryonalen Stammzellen bisher nicht verzichtet werden kann.

Die embryonalen Zellen zeigen, welche molekularen und chemischen Abläufe für die Pluripotenz einer Zelle entscheidend sind und welche Gene für die Pluripotenzeigenschaft aktiviert sein müssen. 
Parallel zur Kultivierung von spermatogonialen Stammzellen ist die Technik zur Erzeugung von induzierten pluripotenten Stammzellen entwickelt worden, wieder zunächst bei der Maus, dann auch am menschlichen Gewebe. Die Grundlage für diese Entwicklung besteht darin, dass jede Körperzelle die gesamte genetische Information des Organismus enthält. Theoretisch kann also jede Körperzelle in eine andere Körperzelle überführt werden und die Funktion einer anderen Körperzelle übernehmen. Wiederum basierend auf den Erkenntnissen an embryonalen Stammzellen, wurde 2007 erstmals die Erzeugung von pluripotenten Zellen aus menschlichen Hautzellen publiziert (10). Hierzu wurden vier verschiedene Gene in die Hautzellen eingeschleust. Im Laufe der vergangenen vier Jahre wurde diese Technik mit geradezu dramatischem Tempo weiterentwickelt. Bevor die induzierten pluripotenten Zellen für regenerative Maßnahmen eingesetzt werden können, sind allerdings weitere Modifikationen der Kultivierungstechnik erforderlich. Für die effiziente Einschleusung der Gene werden nämlich Viren als Transportvehikel benötigt. Mit Viren behandelte Zellen sind aber für den Einsatz am Menschen zu therapeutischen Zwecken nicht geeignet. Es ist aber absehbar, dass eine effiziente und stabile Überführung einer Hautzelle oder einer anderen Körperzelle in eine pluripotente Zelle virusfrei gelingen wird. Auch hier spielen die Erkenntnisse aus der Forschung mit menschlichen embryonalen Stammzellen eine wesentliche Rolle.

\section{iPS Zellen für die Erforschung von Krankheiten}

Auch wenn die induzierten pluripotenten Zellen noch nicht für die regenerative Medizin zur Verfügung stehen, so bieten sie doch bereits jetzt ein immenses Potential für die Erforschung von Krankheiten. Unmittelbare Bedeutung könnten die induzierten pluripotenten Zellen für das Verständnis von angeborenen Erkrankungen und für die Entwicklung neuer Medikamente haben. Werden solche Zellen aus Haut- oder aus Blutzellen von Patienten mit angeborenen genetischen Erkrankungen gewonnen, so kann die Auswirkung der Gendefekte an der ausdifferenzierten Organzelle im Detail im Reagenzglas untersucht werden. Da die Zellen beliebig vermehrbar sind, können an ihnen auch Screeninguntersuchungen von neuen potentiellen Pharmaka eingesetzt werden, mit dem Ziel, eine Beeinflussung der Folgen des Gendefektes zu erreichen. Hierzu ein Beispiel: Es gibt angeborene, lebensbedrohliche Herzrhythmusstörungen durch Mutationen in Genen, 
die für die elektrische Erregungsbildung und Ausbreitung bedeutungsvoll sind. Einige Patienten leiden sowohl an Herzrhythmusstörungen als auch an epileptischen Krampfanfällen, da diese Gene auch die Erregungsabläufe im Gehirn beeinflussen (11). Aus einer Hautzelle eines betroffenen Patienten können sowohl Herz- als auch Nervenzellen hergestellt werden, die dann ebenfalls die Genmutationen enthalten. Solche Zellen können dann elektrophysiologisch untersucht und die Mechanismen der Erkrankung im Herzen und im Gehirn aufgedeckt werden. An ihnen können dann auch ohne Risiken neue Therapieverfahren entwickelt werden.

\section{Zusammenfassung}

Kaum eine Forschungsrichtung unterliegt der Aufmerksamkeit der Öffentlichkeit mehr als die Stammzellforschung. Sie hat das Potential, neue Wege in der Erkenntnis und der Behandlung von Erkrankungen entwickeln zu helfen. Kulturelle, religiöse und gesellschaftliche Normen und die aus ihnen entstehenden gesetzgeberischen Einschränkungen im Umgang mit embryonalen Stammzellen können solche Forschung verhindern. In einer sorgfältigen und ehrlichen Debatte, mit kritischer Abwägung der verschiedenen Standpunkte, muss ein gesellschaftlicher und politischer Konsens gefunden werden. Ein Verbot der Forschung mit embryonalen Stammzellen in der Vergangenheit hätte die Entdeckung von alternativen adulten pluripotenten Zellen verhindert. Bei einer völligen Freigabe hätte sich die Forschung möglicherweise auf die embryonalen Stammzellen fokussiert, und die Alternativen wären nie oder erst viel später entwickelt worden. Im Falle der Stammzellforschnung haben die Reglementierung im Umgang mit embryonalen Stammzellen und die gleichzeitige Förderung der Entwicklung von alternativen Zellen dazu geführt, dass die spermatogoniale Stammzelle und die induzierte pluripotente Zelle entwickelt wurden.

Allgemein gesagt, kann die Gesetzgebung das Grundrecht der Freiheit der Wissenschaft und der Forschung erheblich einschränken. Beispiele sind neben dem Stammzellgesetz das Arzneimittelgesetz, das Medizinproduktegesetz und das Gewebegesetz. Die potentiellen Folgen der Einschränkung der Forschungsfreiheit können für den medizinischen Fortschritt erheblich sein. Gesetzliche Rahmenbedingungen können zusammen mit Fördermaßnahmen aber auch im positiven Sinne die Richtung vorgeben. Hier ist die richtige Entscheidungsfindung im gesellschaftlichen und im politischen Diskurs elementar. Dieser bedarf andererseits einer umfassenden Information der Öffentlichkeit durch die Wissenschaft. 


\section{Literatur}

1. Gesetz zum Schutz von Embryonen (Embryonenschutzgesetz - ESchG) vom 13. Dezember 1990 - Bundesgesetzblatt I S. 2746-2748, 1990.

2. Orlic D, Kajstura J, Chimenti S, Jakoniuk I, Anderson SM, Li B, Pickel J, McKay R, Nadal-Ginard B, Bodine DM, Leri A, Anversa P. Bone marrow cells regenerate infarcted myocardium. Nature 410:701-705, 2001.

3. Murry CE, Soonpaa MH, Reinecke H, Hakajima H, Nakajima HO, Rubart M, Pasumarthi KB, Virag JI, Bartelemez SH, Poppa V, Bradford G, Dowell JD, Williams DA, Field LJ. Haematopoietic stem cells do not transdifferentiate into cardiac myocytes in myocardial infarcts. Nature 428:664-668, 2004.

4. Dressel R, Schindehütte J, Kuhlmann T, Elsner L, Novota P, Baier PC, Schillert A, Bickeböller H, Herrmann T, Trenkwalder C, Paulus W, Mansouri A. The Tumorigenicity of Mouse Embryonic Stem Cells and In Vitro Differentiated Neuronal Cells Is Controlled by the Recipients' Immune Response. PLoS One 3:e2622, 2008.

5. Gottschling C, Albers R, Thielicke R, Sanides S. Reparatur-Set aus dem Embryo. Focus 43:110-117, 2010

6. Thomson JA, Itskovitz-Eldor J, Shapiro SS, Waknitz MA, Swiergiel JJ, Marshall VS, Jones JM. Embryonic stem cell lines derived from human blastocysts. Science 282:1145-1147, 1998.

7. Gesetz zur Sicherstellung des Embryonenschutzes im Zusammenhang mit Einfuhr und Verwendung menschlicher embryonaler Stammzellen - StZG (Stammzellgesetz) vom 28. Juni 2002 - Bundesgesetzblatt I S. 2277 2279, 2002.

8. Guan K, Nayernia K, Maier LS, Wagner S, Dressel R, Lee JH, Nolte J, Wolf F, Li M, Engel W, Hasenfuss G. Pluripotency of spermatogonial stem cells from adult mouse testis. Nature 440:1199-1203, 2006.

9. Guan K, Wagner S, Unsöld B, Maier LS, Kaiser D, Hemmerlein B, Nayernia K, Engel W, Hasenfuss G. Generation of Functional Cardiomyocytes From Adult Mouse Spermatogonial Stem Cells. Circ Res 100:1615-1625, 2007.

10. Takahashi K, Tanabe KI, Ohnuki M, Narita M, Ichisaka T, Tomoda K, Yamanaka S. Induction of Pluripotent Stem Cells from Adult Human Fibroblasts by Defined Factors. Cell 131:1-12, 2007.

11. Lehnart SE, Mongillo M, Bellinger A, Lindegger N, Chen BX, Hsueh W, Reiken S, Wronska A, Drew LJ, Ward CW, Lederer WJ, Kass RS, Morley G, Marks AR. Leaky $\mathrm{Ca} 2+$ release channel/ryanodine receptor 2 causes seizures and sudden cardiac death in mice. J Clin Invest 118:2230-2245, 2008. 